\title{
Examining the Concept of Smart Home Technologies (IoT Systems)
}

\author{
Adjei A. S. Hannah \\ College of Information and Communications Engineering: \\ Southwest University of Science and Technology; Sichuan Province, P. R. CN. \\ serwaa201479@gmail.com
}

\begin{abstract}
With the huge advancement in wireless sensor networks and other computer technologies, Home automation plays an important role in human life by making Lives at home easier and faster. Over the years, several smart home systems have been developed using network wireless systems such as Controller Area Network(CAN), Wi-Fi, Bluetooth, Radio Frequency, Cognitive radio, and several others. With this technology, the user easily controls their homes through their computer and assigns actions dependent on time or sensor readings if any from the device in the network of the home automation irrespective of the location of the user. This technology isn't only limited to controlling appliances around us remotely but also helps in terms of security by warning the user during unusual and critical situations. Home automation is flexibly used in different places with high precision which saves time and money, and also provides comfort, security, and convenience to its user. Home automation can also help the aged and incapacitated people and provide quality of life to people without the need for caregivers or any care from institutions. People's desire for an intelligent home has increased dramatically in recent years as mobile connectivity devices are much more convenient and simple.

This paper focuses on several smart home systems that have been developed in the twentieth century. Networks are enhanced by IoT to collect and analyze data proficiently from sensors and actuators. IoT creating a new era in the world of information and technologies in the areas of security has become vital as intrusion, theft, fire, and leakage of flammable gas that are threats to human lives never fails to continuously be a threat to humanity. IoT is the existing network of the internet where the computer systems will connect to real-world objects or things such as vehicles, devices, home appliances, and many others. This technology saves human energy and it is also a userfriendly safe system that makes the life of its user easier.
\end{abstract}

Keywords: Smart Home, IoT, sensors, home automation ,Controller Area Network.

DOI: $10.7176 /$ ISDE/11-1-06

Publication date: February $29^{\text {th }} 2020$

\section{Introduction}

The interaction between humans and computers is getting to a whole new level as each day goes by in the world today. Cell phones and computers have become a part and parcel of our daily lifestyles as compared to the olden days where computers were highly regarded as tools for managing data and, cellphones as tools for communication. In the world today, there has been an increment of intelligent buildings for the purpose of increasing the safeness and comfortability of its occupants, more homes are being automated by the interconnecting of network electronic equipment [1]. There are several home automation systems on the market today, Home automation using voice recognition, Bluetooth, ZigBee, RFID, DTMF, GSM, IoT, Wi-Fi, and many others. All these technologies operate in either automatic mode or user mode. In the user mode, the controlling is done with instruction given by an android smartphone manually by the user and with the automatic mode, the user can control and monitor appliances in his or her absence remotely [2]. This article focuses on various IoT smart home systems. The IoT can be described as the connection of everyday devices such as smartphones, Internet TV, sensors and actuators with the Internet, whereby the devices are intelligently connected to allow new forms of communication between people. [3]. The Internet has contributed greatly to changing human life by providing connectivity anywhere and anytime with anyone. The Internet helps to bring in an immediate solution for many problems and also enables connectivity from any place within its range which contributes positively to reduce cost and energy consumption. Due to humans desiring for new technology and devices every day, innovators and researchers are always searching and creating new things to satisfy human needs and desires.

\subsection{Problem Statement}

Intrusion, electricity overuse, outbreaks of fire due to electrical fault as well as gas leakages are great disadvantages 
to humanity as far as the security of the home is concerned. Whenever there is a case of theft or a fire incident announced, people sigh relief when the address is given isn't their home or street address. People can't fully concentrate on work or anything when their ward or an elderly loved one of theirs is left home alone, they anxiously call regularly to check up if everything is all right. The pie chart below shows the residential fires in 2017 recorded by the U.S Fire Administration in the United States of America and the cause of it. From the pie chart, it can be observed that the largest percentage of fire outbreaks in 2017 was caused by cooking, unintentional carelessness, open flame, smoking, and electrical malfunctioning. Smart Automation alerts the user should there be any form of unusual activity that is a threat to the safety of the home. [4]

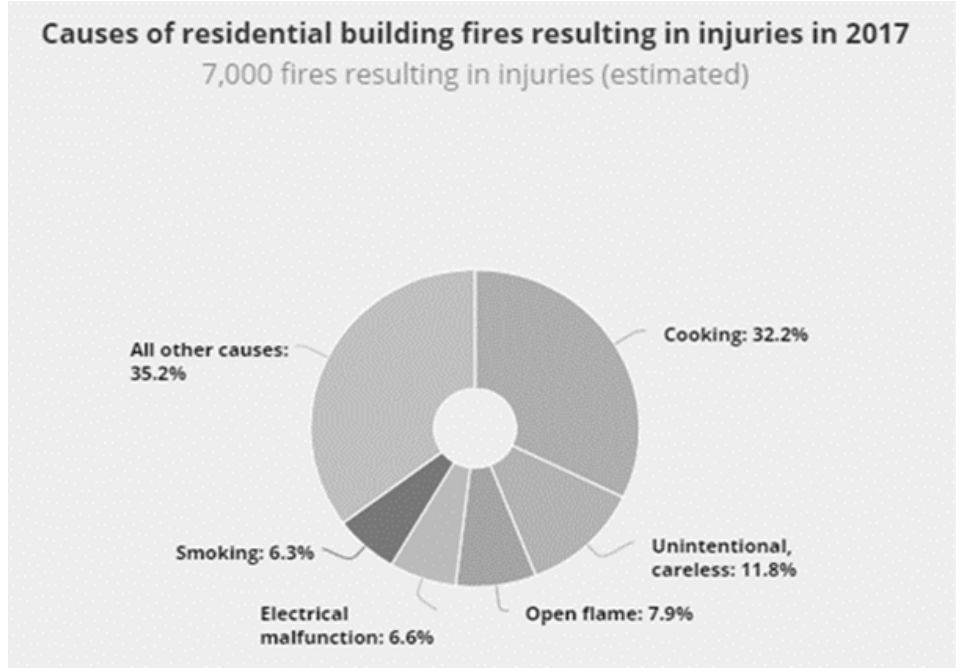

Figure 1.1: Causes of Residential fires in 2017[4]

\subsection{Objective of the Study}

The objective of this paper is to examine and discuss the various concepts of smart home technologies using different systems as well as the importance of using smart home systems.

\subsection{Purpose or the Significance of the Study.}

This study examines different home automation systems that provide better home security and safety, conserves electrical energy, and handles regular household tasks. It explains several systems and how they are implemented to function properly to achieve its major objectives.

\subsection{Literature Review}

\subsection{Related works}

This Chapter discusses some related works of home automation T. R. Gondaliya designed a smart home sensor communication system that provides home automation as well as error detection. The system provides notification to the user should there be any error in the system. This system sends SMS to the service provider about the problem and supports more elasticity, comfort, capacity, and safety. The system designed here uses wireless LAN (Local Area Network) connections between various sensors, hardware modules, and servers as well as various communication protocols between users and servers [6].

S. Kumar designed a ubiquitous smart home system using Android Application. This system is a flexible standalone, low-cost system that is based on an Android app. The system uses an Arduino microcontroller, so no $\mathrm{PC}$ and no other components are required to minimize the cost of the entire system by integrating voice activation for switching Functions. Devices such as light switches, plugs, temperature sensors, humidity sensors, Current sensors, intrusion sensors, smoke/gas sensors, and sirens have been integrated into the system to demonstrate the feasibility and effectiveness of the intelligent proposal system at home. This system successfully performs operations such as switching functions, automatic room control and intruder detection [7]. Manuel et al designed a Controller Area Network (CAN) smart home system. This system has real-time features and ease of implementation and programming of the nodes at a lower cost. This system is mainly used to control the air 
conditioning system and also an intrusion and fire alarm system [1].

Rao et al designed a home automation system using radio frequency to independently control home electrical appliances through radio frequency (RF) either in on state or off state. The control circuit consists of RF transmit and receive modules that are operated at $433 \mathrm{MHz}$ together with a 16f877A PIC microcontroller with peripheral interface control and a few passive components. The system was developed with the PIC16F877a microcontroller. This is the heart and mind of the system. The system is not only inexpensive, but it also offers wide availability, a large customer base and an extensive collection of application notes. The main advantage of this system is that it works with RF technology without a line of sight [8].

\subsection{Internet of Things}

Internet of Things (IoT) refers to the network of everyday objects that often have ubiquitous intelligence. Thanks to the rapid advancement in the underlying technologies, the IoT has opened up a large number of enormous possibilities for a large number of new applications that improve our life's quality. The IoT has described several research technologies and disciplines with which the internet has access to the world of physical objects. The figure below shows the objectives of IoT [8].

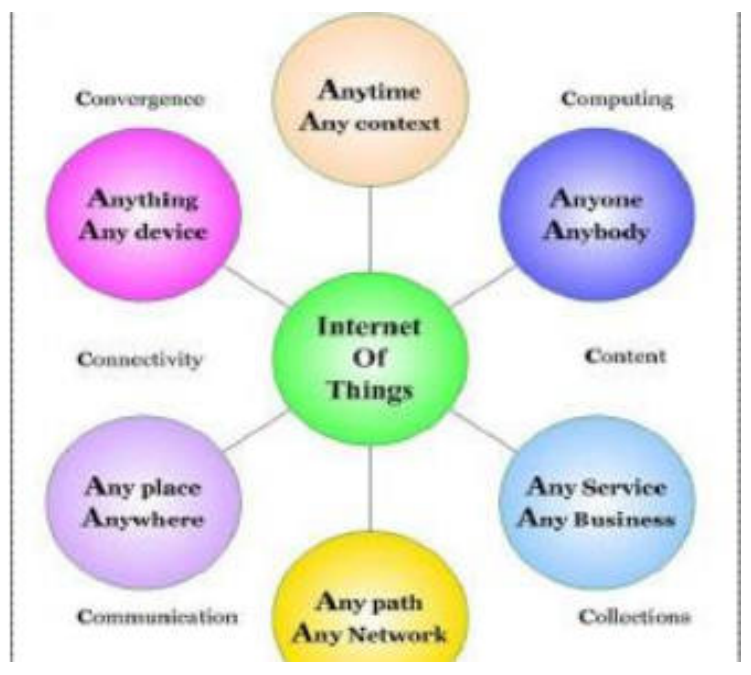

Figure 2.1: Objectives of Internet Things [8].

The concept of IoT though has been in use for quite some years, its concept is gaining much importance with the evolution of advanced wireless technology. The several technologies used to implement IoT in the real world are available. Technology such as Radio Frequency Identification (RFID) is applied to people, objects, and animals and provides a specific address to them. Service technologies such as Machine-to-Machine Communication (M2M), Wireless Sensor and Actuator Networks (WSAN), Vehicle-to-Vehicle communication (V2V), Near Field Communication (NFC) will eventually build up the concept of IoT. Below are several definitions of IoT by different researchers.

According to the China Communication Standards Association (CCSA), the IoT is a network that contains information from the physical world through various devices implemented with the possibility of perception, calculation, execution, and communication, as well as supporting communication between people and things by sending, classifying and collection collects the processing of information [9]

According to ITU (International Telecommunication Union) standards, the Internet of Things is a global infrastructure for the information society that allows advanced services through objects (physical and virtual) based on existing and evolving interoperable information and communication technologies to be connected. In terms of connectivity, ITU-T has created IoT as network connectivity for everyone or anything, anytime, anywhere [10].

According to Chen et al. Is IoT "an intelligent network that connects everything to the Internet to exchange information and to communicate with devices to collect information according to the agreed protocols". The IoT was also created as an extension of the interaction between applications and people from a very naive perspective of "things" for communication. "Things" can be treated as a combination of hardware, software, data, and services 
[11].

\section{Architecture of the Internet of Things}

Although there is no general structure for IoT, Different researchers have proposed models of different architecture. Below is a figure that shows it [12].

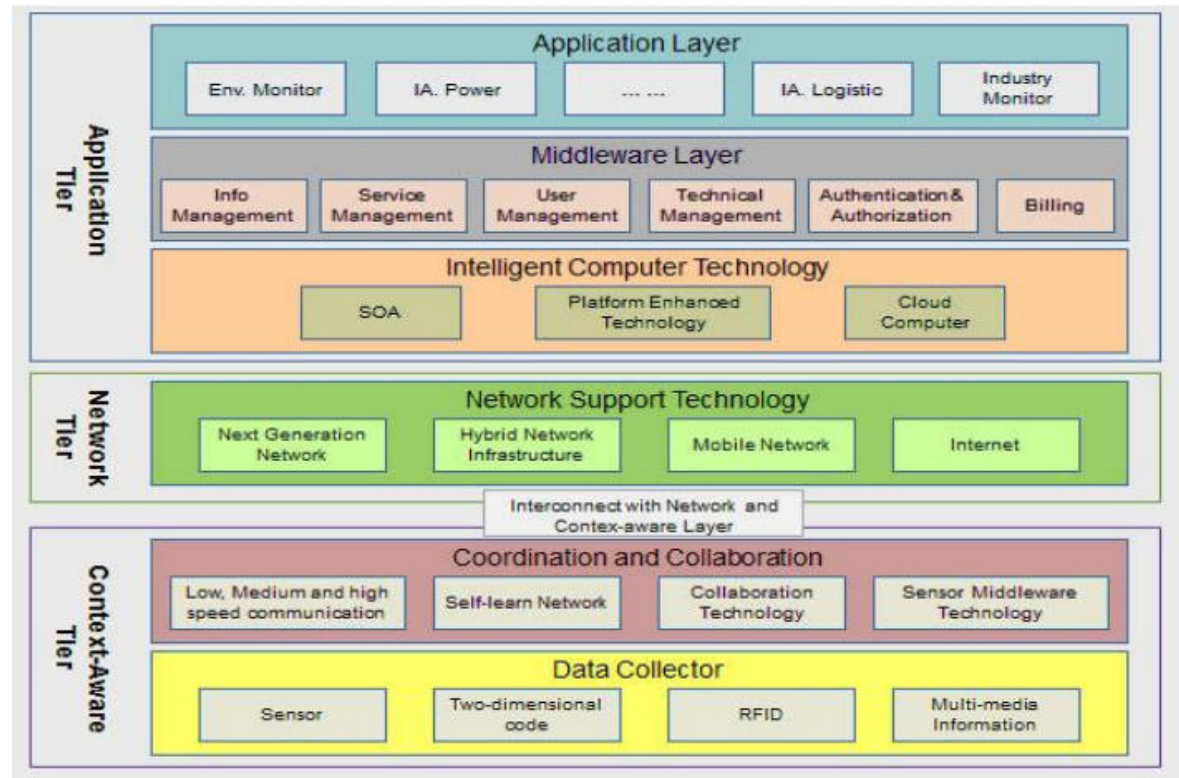

Figure 2.2: Architecture of IoT[12].

\section{Applications of IoT}

IoT is applied in different aspects of life. IoT has been grouped into domestic, societal, environmental, technological and emergency and critical situation applications. The figure below shows the categories and subcategories of the IoT application domain.

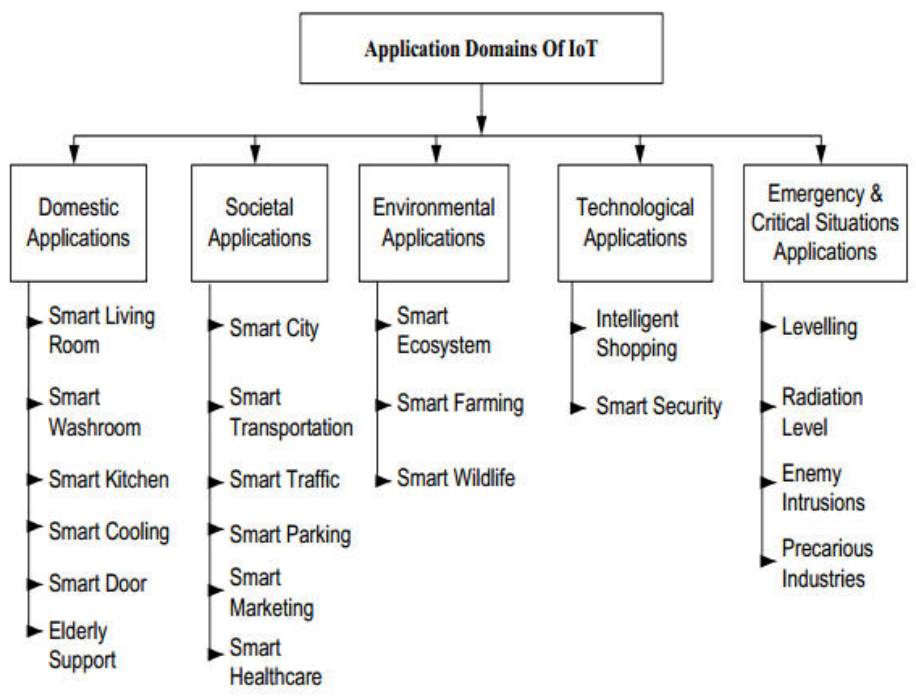

Figure 2.3: Applications of IoT [13] 


\subsection{Smart Home}

\subsection{Smart Home and its Features}

Home Automation known as smart home is a technology used in the home to provide security, convenience, and comfort to its user. Using smart home internet of things, different wireless technologies that support remote data transfer, control and sensing systems such as RFID, Wi-Fi, Bluetooth, and cellular networks have been evolved to add intelligence at various levels in the homes [14]. Smart home makes the life of its users much easier and safer at home. With a smart home, individual finds security knowing that should there be any intrusion or fire cases while they sleep or are away from home, their system at home will alert them and necessary measures will be taken. Smart home not only gives security but also gives its user a feeling of relief especially if the user has a disabled or an elderly family member at home, the user can go to work with little or no anxiety knowing very well that his or dear one is in a safe home and can fully focus on his her work with no distractions of calling home every hour to check up on his or her loved one.

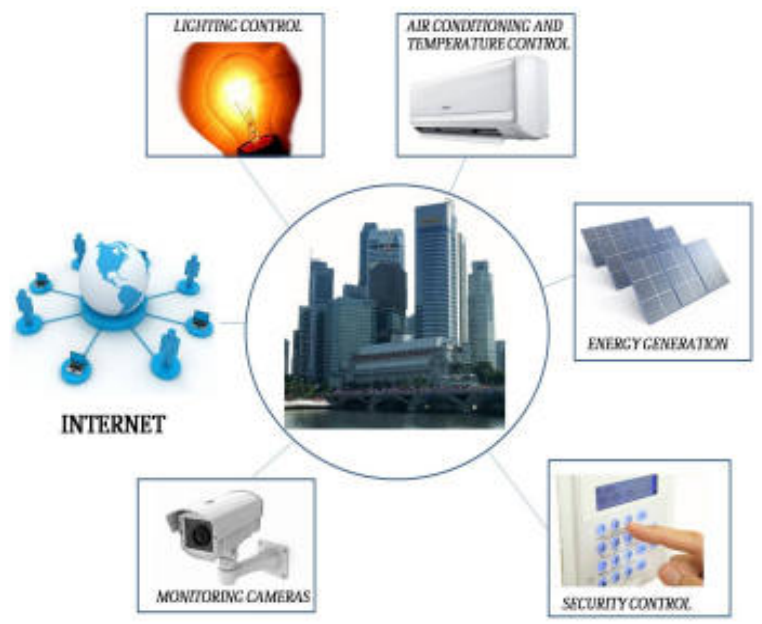

Figure 3.1: Smart Home.

\subsection{Why are Internet of Things needed at the Home?}

IoT proposes new services to make the home smarter, improving the communication between users and devices and hence improving the quality, safety, and easiness of life at home.

\subsection{Challenges of IoT as related to SMART HOME}

In as much as IoT is a technology used a lot today to improve the life of its users, IoT has several challenges. However, these challenges according to Matta et al, are categorized into three areas namely technological challenges, societal challenges, and environmental challenges.

\section{Technological Challenges:}

Technological challenges are subdivided into Architecture and Heterogeneity, resource management, efficient data handling, and security and privacy. The architecture aims at the interconnection of the Wireless Sensor Actuator Network (WSAN) to the internet as a web service. These services include door entry control which grants access to an authenticated person. Heterogeneity manages all devices, various network topologies, network configuration and various forms of data representation. It is challenging to support heterogeneity efficiently so that all devices can be managed properly. The challenge of resource management lies in where and how resources should be well planned and managed efficiently. Resources comprise of soft agents, smart objects, sensors as well as actuators that communicate with each other to utilize the paradigm of IoT to its fullest and efficient handling of data which may be a transformed, generated or stored data in the transit. Data collection forms a major challenge and one of its major problems is in the field and realization of IoT is "Data exchange among large-scale heterogeneous network elements". Security and privacy during the sensing of data is also a big challenge in IoT [13]. 


\section{Societal challenges:}

Some common societal challenges of IoT are talent breach, disapproval of the novel paradigm by masses, userfriendliness and many others [13].

\section{Environmental challenges:}

Here challenges such as power consumption, failure tolerance, and economy concerning cost are some of the major environmental challenges of IoT [13].

\subsection{Suggested Solutions to Overcome the Challenges}

$>$ A large storage database is needed to store all data collected.

$>$ System should take appropriate measures on security threats and also do reconfiguration after an attack.

$>$ Servers should be configured in a way that it will give access to only authenticated users.

$>$ Big Data analytics and algorithm can be useful in tackling the issue of talent breach

$>$ Work on implementing low power consumption systems

\section{Discussion}

From the different IoT systems discussed above, one can understand that IoT is an important technology that makes its users' life easier and livelier, although all the IoT systems discussed above achieves its primary objectives, I strongly suggest an IoT system that uses a microcontroller (Arduino). This is because when designing a system, one should consider the cost, portability, scalability, power consumption, whether or not the device used can be integrated into the system and the most important of them all, how user-friendly it is. The use of Arduino eliminates the need of personal computers as it becomes the heart and the brain of the system, it becomes the main controller that hosts all others sensors and performs the necessary actions needed to be done for the smooth operation of the system. Arduino microcontroller is an open-source device which is portable, low cost, and very user-friendly that is, its software runs on Windows, Linux, and Macintosh OSX operating systems and has a clear and a simple programming environment for both beginners and advanced users to make good use of due to its flexibility. Arduino microcontrollers can be programmed so that user authentication will be required to access the smart home which gives it a major advantage as far as security is concerned. There are several types of Arduino microcontrollers, types such as Arduino Mega 2560, Arduino ATmega8U2, Arduino ATmega328p and many others that a user can select from depending on the features needed to ensure an efficient and successful system. Below is a figure that demonstrates the use of Arduino microcontrollers in designing a smart home system by S. Kumar.

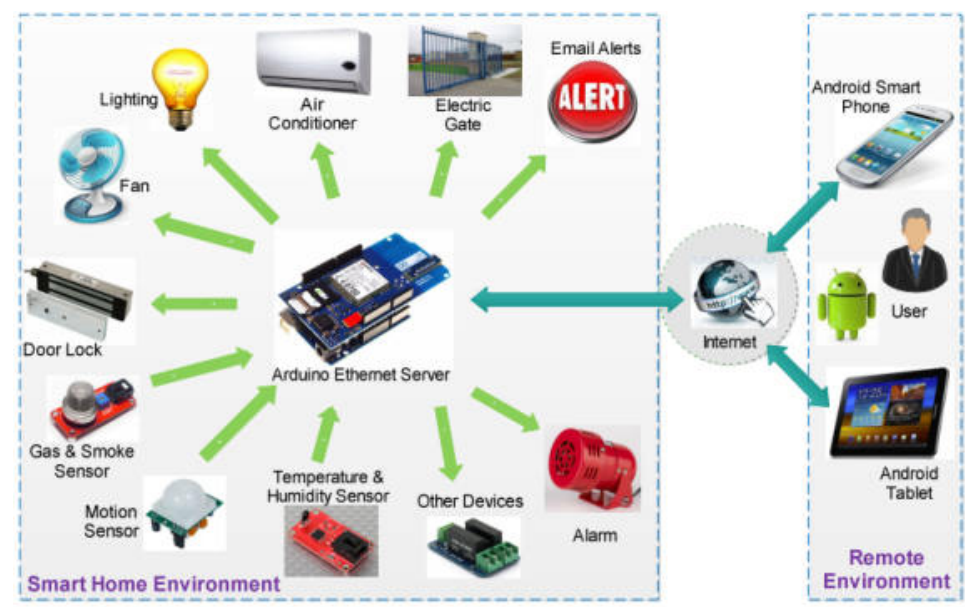

Figure 4.1: A Smart home System using Arduino [6] 


\section{1 Recommendation}

It is recommended that future works of similar papers should include components like cameras and incorporating SMS as well as call alerts to enhance smart automation device's quality and efficiency.

\section{Conclusions}

In this paper, several home automation systems have been discussed. The systems discussed here work satisfactorily and the appliances connected to it were successfully controlled from either the computer, mobile device or whichever platform used. The several papers discussed above all provides convenience to its users, helps the elderly and the disabled, provides security and comfort as well as makes the user's life easier at home or even outside the home. The papers have met all general objectives. The systems support monitoring enhances mobility and supports the control of devices from any remote location within the range of the wireless communication technology used. The above systems can also be improved upon for higher work efficiency.

\section{References}

[1]. M. Ortiz, M. Diaz, F. Bellido, E. Saez and F. Quiles, "Smart home automation using Controller Area Netwok", Advances in Intelligent and soft Computing, 2011.

[2]. D. Pooja J, Y. V. Lathkar, D. B. Purushottam, "Smart home using Android Application” IJRET, vol. 3, pp. 365-367,2014...

[3]. G. Kortuem, F. Kawsar, D. Fitton, and V. Sundramoorthy, "Smart objects as building blocks for the internet of things," Internet Computing, IEEE, vol. 14, pp. 44-51, 2010.

[4] usfa.fema.gov/data/statistics," Causes of Residential building fires", 2017...

[5] T.R. Gondaliya,"Sensor communications in smart home", International Institution for Technological Research and development, vol. 1, Issue 3, 2016.

[6] S. Kumar, "Ubiquitous smart Home System Using Android Application”, IJCNC, vol.6, No. 1, pp $33-43,2014$

[7] Y. L. Rao, P H. Babu, V. Prasad, T. S. Rao, “home automation using radio frequency”, IJEETE, vol. 03, Issue. 4, pp. 259-263 2016.

[8] G. Tripathi, D. Singh and A. J. Jara, “A survey of Internet-of-Things: Future vision, architecture, Challenges and services", IEEE World Forum on Internet of Things (WF-IoT), pp. 287-292, 2014.

[9].China Communications Standards Association.(2011), \{YDB $\}$ Communication standard technical report. Retrieved March 20, 2017, from http://www.ccsa.org.cn/english/list std.php?.tbname=ydb doc\&keyword=\&page_currentPage=4.

[10] International Telecommunication Union (ITU). (2012). Y.2060: Overview of IoT. Retrieved January 15, 2017, from https://www.itu.int/rec/T-REC-Y.2060- 201206-I

[11] Chen, S.; Xu, H.; Liu, D.; Hu, B.; and Wang, H. (2014). A vision of IoT: Applications, challenges, opportunities with China perspective. IEEE Internet of Things Journal, 1(4), 349-359.

[12] J. F. Abu-Saif “ Internet of Things” E- Technologies, student Information system, PPU, 2015

[13] S. D. T Kelly, N. K. Suryadevara, S. C. Mukhopadhyay, "towards the Implementation of IoT for Environmental Condition Monitoring in Homes”, IEEE, vol.13, pp. 3846-3853, 2013.

[14] P. Matta, B. Pant, "Internet of Things: Genesis, challenges and applications", Journal of Engineering Science and Technology, vol. 14, No.3, pp. 1717-1750, 2019. 